\title{
Knowledge Attitude and Practice of Body Donation in Medical Students: A descriptive Study
}

Dr. Ajay $\mathrm{N}^{1}$, Dr. Asharani $\mathrm{SK}^{2 *}$, Dr. Tejaswi $\mathrm{HL}^{3}$

${ }^{1}$ Assistant Professor, ${ }^{2}$ Associate Professor, ${ }^{3}$ Associate Professor, Department of Anatomy Adichunchanagiri Institute of Medical Sciences, B G Nagara, Nagamangala (Taluk) Mandya (district) Karnataka, (State), India

DOI: $\underline{10.36348 / \text { sijap.2020.v03i11.002 }}$

| Received: 15.11.2020 | Accepted: 26.11.2020 | Published: 30.11 .2020

*Corresponding author: Dr. Asharani SK

Abstract

The aim of the study was to ascertain the knowledge attitude and practices of Indian medical students towards post mortem body donation. An online questionnaire was sent to 150 students who have witnessed and performed human cadaver dissection. Even though most of the respondents are aware of the existence of the practice of body donation (98\% aware) and are direct beneficiaries of it, a substantial number of them lack an understanding of the laws governing body donation (only 32.7\% are aware of the Indian Anatomy Act, 1948 and 26.5\% of the Karnataka Anatomy Act, 1957?) and a majority are hesitant to donate their own body for medical research and learning (46.9\% are willing to donate). The reason for unwillingness to donate their body could be stemming from hesitation to be the first one in their peer/family circle to pledge to donate ( $87.8 \%$ have no one close to them who has donated), an apprehension that their body will be ill-treated (26.5\%), opposition from family/friends $(61.2 \%)$ and religious beliefs $(53.1 \%)$.

Keywords: Body donation, cadaver, anatomy, medical education, medical research, teaching.

Copyright (C) 2020 The Author(s): This is an open-access article distributed under the terms of the Creative Commons Attribution 4.0 International License (CC BY-NC 4.0) which permits unrestricted use, distribution, and reproduction in any medium for non-commercial use provided the original author and source are credited.

\section{INTRODUCTION}

It is broadly recognized in the scientific literature that cadaveric dissection is an integral part of medical teaching and research [1]. The experiences in a cadaver lab has a profound influence on a student's attitude and behavior in a clinical setting and with patients [2]. Historically, in India, though body donation was legal, unclaimed dead bodies were the main source of dissection cadavers to meet the demands of a few medical colleges that existed. Owing to the increase in the number of medical colleges and undergraduate and post graduate seats, a severe shortage in the availability of cadavers has arisen, which has necessitated a need to popularize the body donation practice among the general population as well as in medicos who, as a group, are the direct beneficiaries of such donations [2, 3]. This study attempts to gauge the knowledge, attitudes and practices of medical students of India towards body donation for medical teaching and research and provide a background for planning of programmes to increase awareness and willingness towards body donation.

\section{EXPERIMENTAL SECTION}

This study is a survey based descriptive study. Sampling method used is a type of non-probability sampling; convenience sampling. Institutional ethical committee permission sought. A semi-structured, pretested and validated questionnaire was given to 145 medical students of Adichunchanagiri Institute of Medical Sciences, B G Nagara, Karnataka, India. Results were tabulated in Microsoft .xlsx file format and statistical data points were represented in graphical form and analysed.

\section{RESULTS}

An overwhelming majority of the respondents $(98 \%)$ are aware of body donation for medical teaching and research purposes.

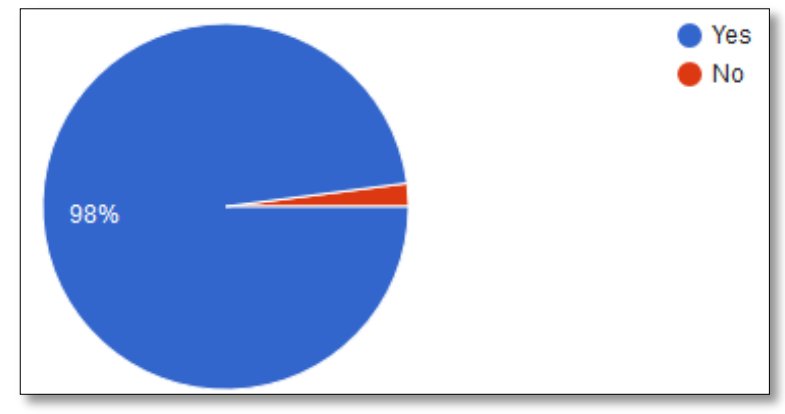

Fig-1: Are you aware of body donation for teaching and research purposes? 
However, a substantial number of them lack an understanding of the laws governing body donation (only $32.7 \%$ are aware of the Indian Anatomy Act, 1948 and 26.5\% of the Karnataka Anatomy Act, 1957).

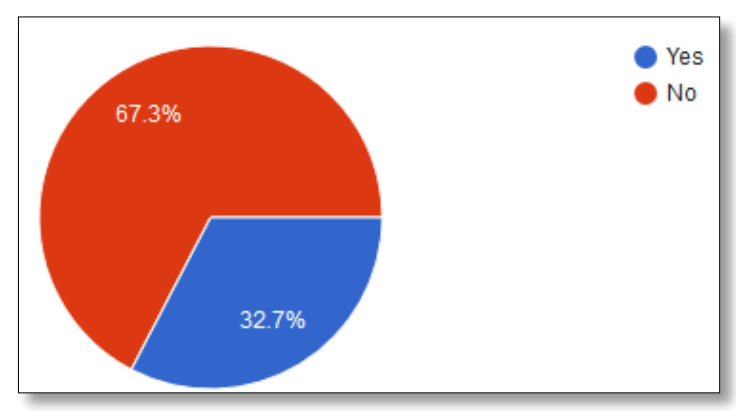

Fig-2: Are you aware of the Indian Anatomy Act, 1948?

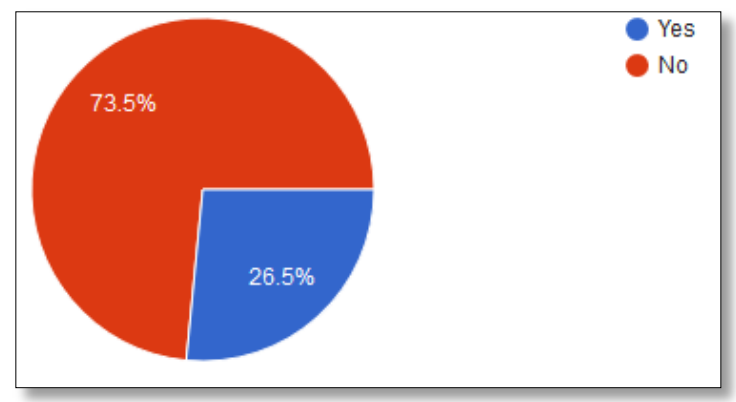

Fig-3: Are you aware of The Karnataka Anatomy Act, 1957?

A majority are hesitant to donate their own body for medical research and learning (only $46.9 \%$ willing to donate), and only $8.2 \%$ have already pledged for body donation.

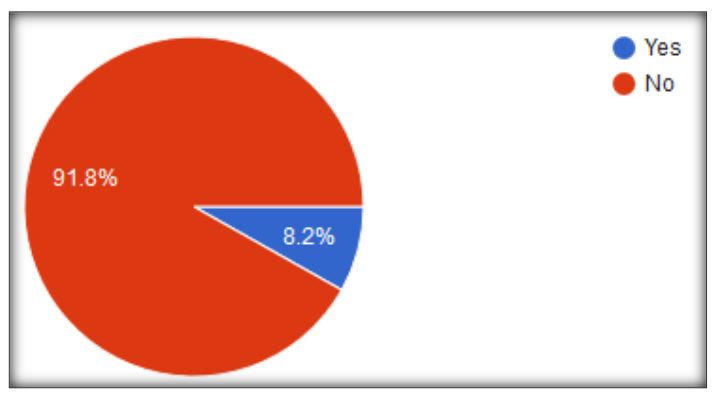

Fig-4: Have you pledged/signed up for body donation?

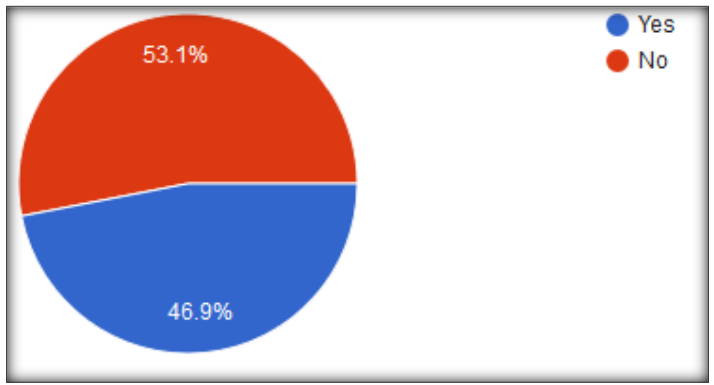

Fig-5: Are you inclined to donate your body when you die?

However $67.3 \%$ of them have motivated their family members and acquaintances towards donation.

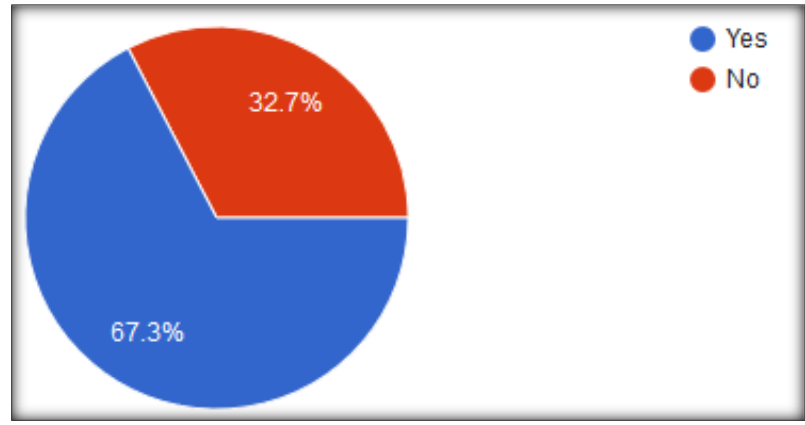

Fig-6: Do you motivate your family members/ acquaintances towards body donation?

The reason for unwillingness to donate their body may be stemming from hesitation to be the first one in their peer/family circle to pledge to donate ( $87.8 \%$ have no one close to them who has donated), an apprehension that their body will be ill-treated (26.5\%), opposition from family/friends $(61.2 \%)$ and religious beliefs $(53.1 \%)$.

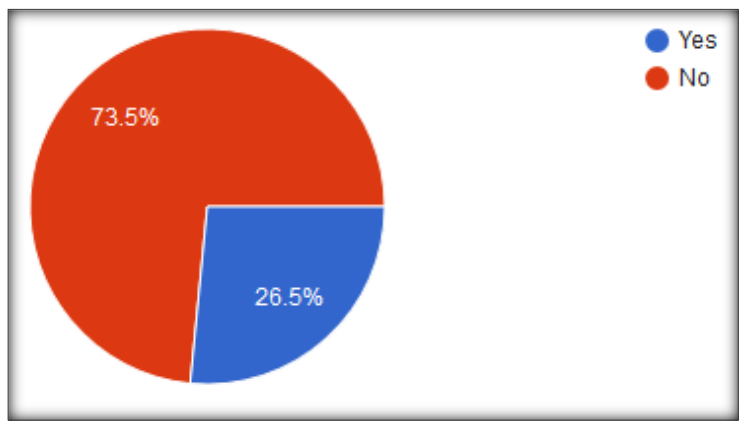

Fig-7: Are you apprehensive that your body will be ill-treated (disfigurement, disrespect etc.) if you choose to donate?

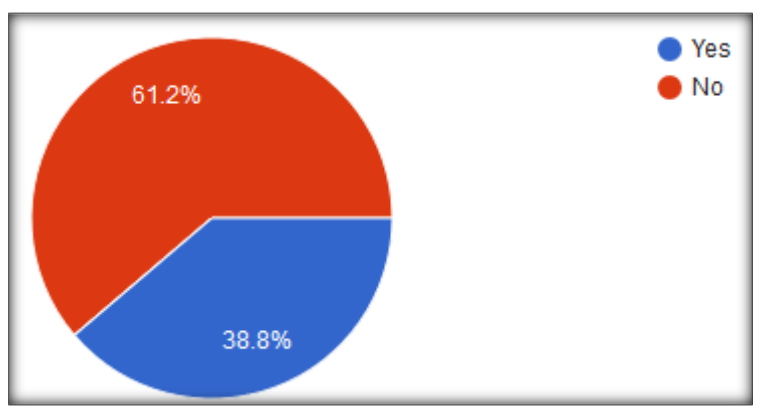

Fig-8: Will your family/friends be supportive of your decision of body donation?

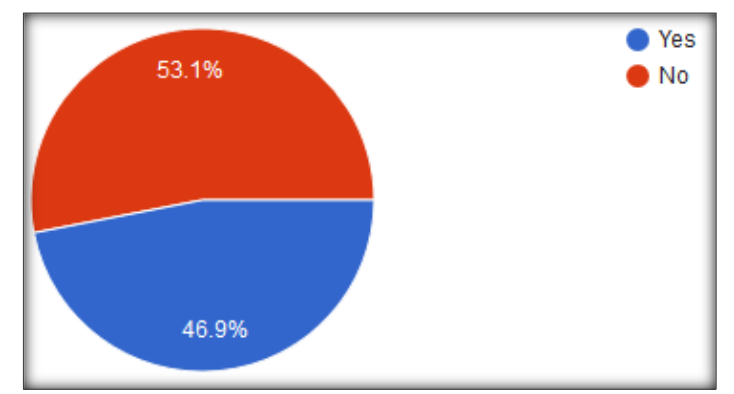

Fig-9: Do your religious beliefs support body donation? 


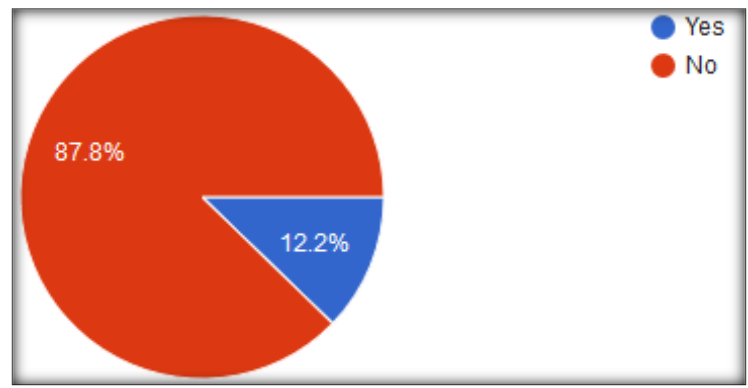

Fig-10: Has anybody in your family donated their body?

\section{DISCUSSION}

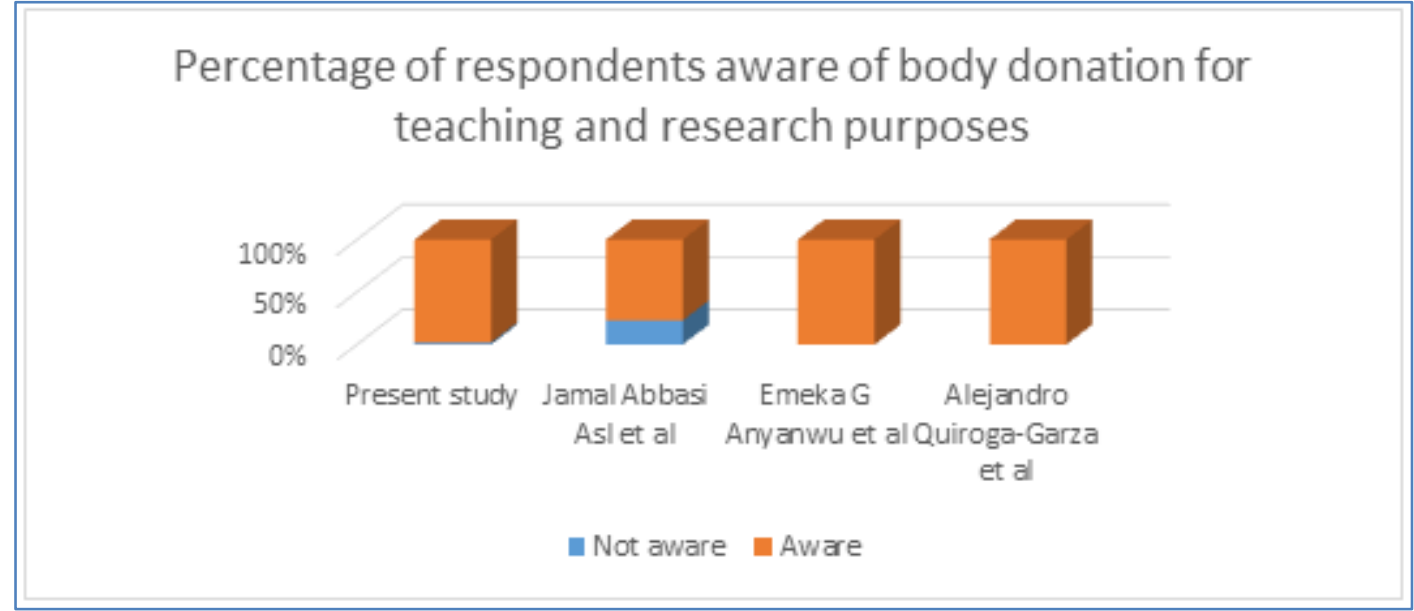

As demonstrated by other studies; Jamal et al. [4], Emeka et al. [5] and Alejandro et al. [7], the present study too shows a very high degree of awareness towards body donation for medical teaching and research purposes.

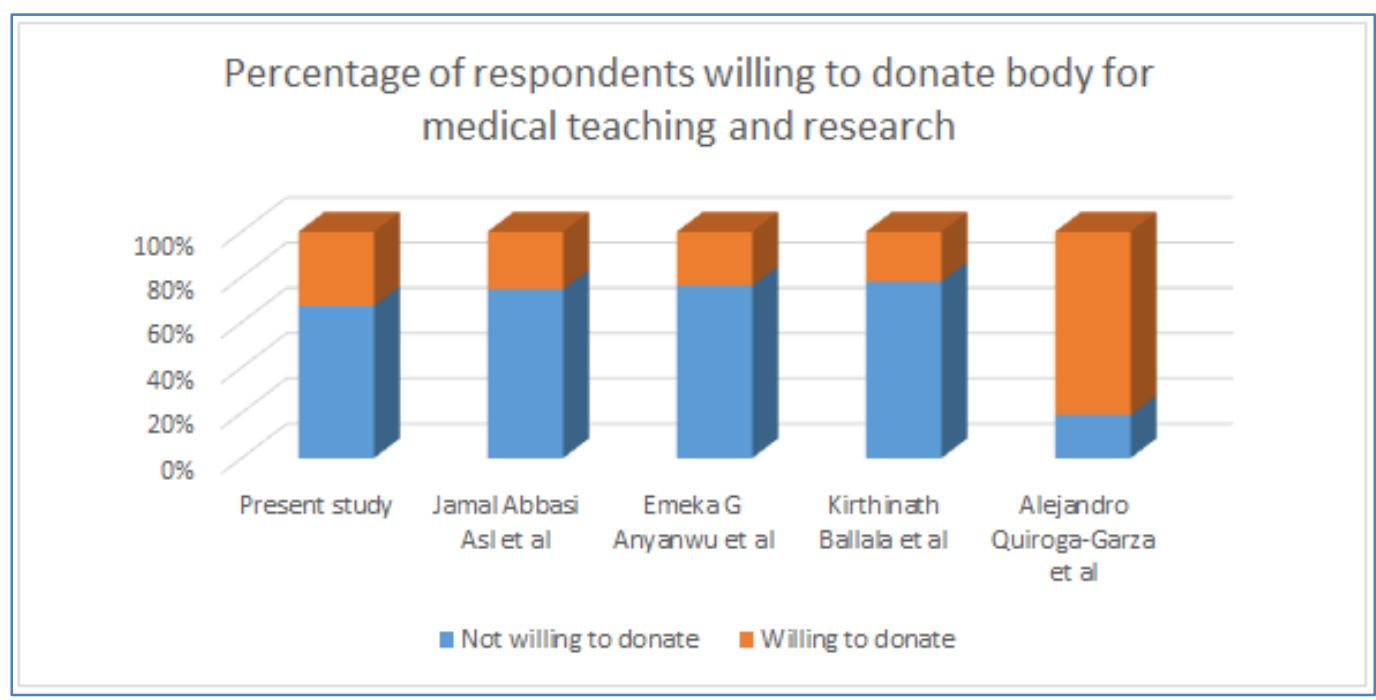

However there is a sharp change in the opinion of the respondents regarding willingness to donate one's own body according to Jamal et al. [4], Emeka et al. [5] and Kirthinath B et al. [6].

The present study is in agreement with these studies. In a study conducted by Alejandro et al. [7] in Mexico though, a very high percentage of willingness was recorded from the respondents and the percentage of willingness increased after exposure to dissection.

Michelle et al. [8] found in their study of Australian chiropractic students that atheists and agnostics were more willing to donate compared to respondents who declared they to be religious. This is reflected in the present study with $53.1 \%$ saying that 
their religion doesn't support body donation. Michelle et al. [8] and Emeka et al. [9] also observed that subjects exposed to dissection tended to change their attitude towards body donation with more subjects becoming unwilling to donate. Emeka et al. [9] qualifies the exposure based on duration and states that limited exposure to dissection and mere observation caused a decrease in willingness to donate, whereas prolonged exposure and performance of dissection caused a reversal of opinion with respondents becoming more willing to donate. Emeka et al. [9] also noted that unwillingness to donate was linked to perceived illtreatment of cadavers in dissection lab settings.

Jamal et al. [4] noted that lack of awareness was the major reason for unwillingness to donate where as Emeka G et al. [5] recorded that family opposition was the most common reason; Kirthinath B et al. [6] observed that fear of misuse to the body was the primary reason for unwillingness to donate. In the present study, respondents gave the following reasons for unwillingness to donate; apprehension that their body will be ill-treated (26.5\%), opposition from family/friends $(61.2 \%)$ and religious beliefs $(53.1 \%)$. Kirthinath B et al. [6] recorded that only $7 \%$ of the respondents had already registered for body donation.

Medical and allied students and professionals, though being the primary beneficiaries of body donation are hesitant to donate their own body or their loved ones' for medical research and teaching. This attitude may be prevalent due to their religious beliefs or socio-cultural practices or acquired due to their experiences in observation and handling of cadavers in the dissection laboratory. It is imperative that medical professionals are encouraged to donate their bodies by working with community leaders and religious heads and creating awareness regarding the benefits of body donation. Allaying fears of misuse or ill treatment of cadavers by establishing and following strict protocols in cadaver handling in labs is also important. This will go a long way in assuring the donors that their mortal remains will be treated with respect and will be only used for the purpose they intended.

\section{CONCLUSION}

Medical and allied students and professionals are highly aware of body donation for medical teaching and research. However their willingness to donate is very is low. Major reasons attributed to unwillingness are they have no one close to them who has donated, an apprehension that their body will be ill-treated, opposition from family/friends and religious beliefs.
Efforts must be undertaken to increase awareness and motivate medical professionals to donate their bodies for medical education.

\section{REFERENCES}

1. Rokade, S. A., \& Bahetee, B. H. (2013). Body donation in India: a review. International journal of research in medical sciences, 1(3), 173-177.

2. Ciliberti, R., Gulino, M., Gazzaniga, V., Gallo, F., Vellone, V. G., De Stefano, F., ... \& Baldelli, I. (2018). A survey on the knowledge and attitudes of Italian medical students toward body donation: Ethical and scientific considerations. Journal of clinical medicine, 7(7), 168.

3. Karmakar, N., Chakraborty, T., Datta, A., Nag, K., Das, S., \& Bhattacharjee, P. (2020). Knowledge, attitude, and practice regarding voluntary wholebody donation among medicos in Northeast India. CHRISMED Journal of Health and Research, 7(2), 103.

4. Abbasi Asl, J., Nikzad, H., Taherian, A., Atlasi, M. A., Naderian, H., Mousavi, G., ... \& Omidi, A. (2017). Cultural acceptability and personal willingness of Iranian students toward cadaveric donation. Anatomical sciences education, 10(2), 120-126.

5. Anyanwu, E. G., \& Obikili, E. N. (2012). Dissecting the dissectors: Knowledge, attitude, and practice of body bequests by Nigerian anatomists. Anatomical Sciences Education, 5(6), 347-353.

6. Ballala, K., Shetty, A., \& Malpe, S. B. (2011). Knowledge, attitude, and practices regarding whole body donation among medical professionals in a hospital in India. Anatomical sciences education, 4(3), 142-150.

7. Quiroga- Garza, A., Reyes- Hernández, C. G., Zarate- Garza, P. P., Esparza- Hernández, C. N., Gutierrez- de la O, J., de la Fuente- Villarreal, D., ... \& Guzman- Lopez, S. (2017). Willingness toward organ and body donation among anatomy professors and students in Mexico. Anatomical sciences education, 10(6), 589-597.

8. Alexander, M., Marten, M., Stewart, E., Serafin, S., \& Štrkalj, G. (2014). Attitudes of Australian chiropractic students toward whole body donation: A cross- sectional study. Anatomical sciences education, 7(2), 117-123.

9. Anyanwu, E. G., Obikili, E. N., \& Agu, A. U. (2014). The dissection room experience: A factor in the choice of organ and whole body donationA Nigerian survey. Anatomical sciences education, 7(1), 56-63. 